\title{
Collapse of the Ontological Gradient
}

\section{Ted Dace}

An independent scholar and author of Escape from Quantopia (Iff Books, 2014)

(Kansas City, Missouri, USA)

E-mail: tdace@protonmail.com

https://orcid.org/0000-0002-4600-0650

\begin{abstract}
Because an unmeasured quantum system consists of information - neither tangible existence nor its complete absence - no property can be assigned a definite value, only a range of likely values should it be measured. The instantaneous transition from information to matter establishes a gradient between being and not-being. A quantum system enters a determinate state in a particular moment (being) until this moment is past (not-being), at which point the system resumes its default state as an evolving superposition of potential values of properties, neither strictly being nor not-being. Like a "self-organized" chemical system that derives energy from breaking down environmental gradients, a quantum system derives information from breaking down the ontological gradient. An organism is a body in the context of energy and a mind in the context of information.

Keywords: Mind-body problem, Measurement problem, Complementarity, Implicate order, Memory, Nonequilibrium thermodynamics
\end{abstract}

Received: July 23, 2019; accepted: August 28, 2019

Philosophy and Cosmology, Volume 24, 2020: 70-82.

https://doi.org/10.29202/phil-cosm/24/8

\section{Introduction}

Ever since Descartes characterized matter and mind in terms of extensive and cogitative substances, philosophers and scientists have struggled to resolve the mind-body problem. How do fundamentally different "substances" collaborate in the seamless workings of the brain? Surely, there is ultimately one substance only. Yet the materialist reduction of mind to brain makes no more sense than the idealist reduction of brain to mind. As Bergson pointed out (Bergson, 1911: 10-11), the brain cannot be at once a center of physical activity and a seat of representations of physical activity. A material object is bound by the principle of identity: $A=A$. Representational thought, on the other hand, entails $A=B$ or $C$ or $D$, etc., depending on what object $A$ is said to represent, such as a building or a car or a desk. To reduce mind to brain — to claim that the mind in reality is only the brain at work - is to suspend the principle of identity in the case of neurotransmission. Yet the brain, unless it has a magical property of intentionality or "aboutness," remains a material object like any other — albeit a highly complex material object — and therefore cannot contain any mental properties even where its activities clearly correlate with them.

(C) Dace, Ted, 2020 
The failure to resolve the mind-body problem allowed it to fester and mutate and metastasize until it was the central dilemma of physics: the quantum-mechanical measurement problem. According to the measurement postulate — a way of interpreting the famously "strange" rules of quantum mechanics - to transform the indefinite values of the properties of an isolated microphysical system into definite values requires a measurement, which means an interaction, a happening. In essence, what is postulated is time-passage. In order for us to have the sensation of tangible objects, atoms must be endowed with definite properties, not evolving sets of possibilities in superposition, as in the domain of Schrödinger's wave mechanics.

The problem with the measurement postulate, which was proposed by Dirac and developed by von Neumann (Whitaker, 2006: 195), is that classical measurement uncovers values rather than creating them. By contrast, instead of just telling us what the electron is, quantum measurement calls forth from a cloud of indeterminacy a suitable set of results, though it could just as easily call forth different results so long as they fall within parameters set by a mathematical procedure known as the wave function. Why does an event, including the event of a measurement, endow with definitude a quantum system otherwise characterized by the systematic development - given the forces acting on it — of various possible configurations?

Since quantum mechanics is fundamental and classical mechanics only approximate, we might ask why there is a classical world at all. Where is the divide or "cut" between the inherently indeterminate system under investigation and the ostensibly classical device by which we observe it? A measuring device is composed of atoms, all of which are described by wave functions, so why not assign a wave function to the measuring device as a whole? Though the rules of quantum mechanics typically manifest only in the very small, in theory they ought to apply at all scales. What prevents "infection" of the measuring apparatus with the indefiniteness - the superposition of many values for each property - that characterizes the quantum system prior to measurement? To put it another way, what magical property enables the measuring device to force a classical outcome for the system under investigation?

"Measuring devices," writes Peter J. Lewis, "are just hunks of matter, obeying the same physical laws as any other hunk of matter" (Lewis, 2016: 50). The same could be said of the brain.

A quantum measuring device might include a microscope through which an observer views a mark left by an atom on a photographic plate. Nothing fundamental divides the atom from that mark or, for that matter, from the microscope or the observer's eye or the optic nerve that leads to the occipital lobe of the observer's cerebrum. As far as von Neumann could see, the only cut was between the brain and consciousness (Whitaker, 2006: 173, 198). The quantum dilemma is the mind-body problem transposed onto physics.

According to Bergson (1911: 293-95), Descartes' mistake was to contrast thought with the spatial property of extension. Bergson approached the issue in terms of time. Starting with the act of perception, which is neither purely material like sensation nor purely mental like thought, as we move in the direction of sensation and matter we ultimately arrive at a sequence of discrete moments, each identical to the previous except for the rearrangement of atoms in space. By contrast, as we move in the direction of thought we find memory, the preservation of the past as a function of indivisible duration. In one direction time is pulverized into durationless instants, each of which instantaneously converts from present to past, a sort of parade into oblivion. In the other direction time is a single movement with nothing demarcating past from present.

Memory is the inescapable outcome of time as flux. As a material object the brain can no more represent the past, i.e. that which is no longer there, than that which is. Only in the 
context of continuous time - in particular the ongoing presence we call consciousness - is there representation. Matter, on the other hand, is limited to mere presentation.

Seeking a framework within which research could be conducted even without a clear description of the underlying reality of the quantum system, Bohr developed the principle of complementarity in conjunction with Heisenberg's discovery of the uncertainty principle, that is, the impossibility of obtaining precise values of position and momentum in the same measurement (Whitaker, 2006: 176). Though long regarded as inherently perplexing, in the proper temporal context complementarity is a model of simplicity. Implicit in a definite position is a distinct moment. Thus, a particle — say, a photon — has a precise position at a particular time. Momentum, on the other hand, is not limited to any given moment but carries over from before. Where position is a snapshot - a single instant in a sequence of instants - momentum is the current that runs through them, converting snapshots into cinema. Like momentum and position, energy and instantaneous time are also conjugate variables. As Whitehead observed, a vibratory system has no existence as such at less than a certain duration (Capek, 1971: 318). Since the value of energy is by necessity uncertain at an instant, to arrive at a precise energy level can only mean a degree of uncertainty in the timing.

Einstein came into the 1927 Solvay Conference with the intention of proving that position and momentum (and time and energy) can be determined in the same measurement. He was soon compelled to concede the point to Bohr (Whitaker, 2006: 205-09). However, Bohr could offer no fundamental explanation for why Einstein's seemingly sensible approach was faulty. Bergson might have pointed out that time cannot be revealed as both continuous flow and a sequence of discrete moments at the same time. Either time is flux or the moments abstracted from it. As it depends on context, we can look at it either way. The genius of complementarity is the recognition that the contradiction is in our perspective, not the actuality.

\section{The Instantiation Postulate}

Quantum mechanics combines two approaches to the study of microphysical systems. Whereas the fundamental dynamics are expressed in the Schrödinger equation - the solution of which for any given set of boundary conditions is the wave function - a measurement generates a definite value for the measured property. That the Schrödinger equation is linear means each superposition of values of a given property encoded in the wave function can be succeeded only by another superposition of values. To get from wave mechanics to the classical mechanics of large-scale sensorial existence, we need a nonlinear dynamic which, like a limb projected from a tree trunk, would allow for a departure from superposition into a single definite value of each property of the system under investigation. In the absence of any law or equation for such an outcome, the result of a measurement of, say, the spin of an electron ought to be a superposition of the measuring device indicating "spin up" and "spin down." Since we know the actual measurement generates one result or the other rather than both, we naturally conclude that quantum theory is wrong or at least missing something (Albert, 2015: 161).

Einstein began his commentary at Solvay with the claim that quantum theory is incomplete. How can an electron be in a superposition of indeterminate states while on its way to a photographic plate only to reveal definite values of properties when it reaches the plate? Surely it possessed those values during the entire journey and not just at the moment of arrival (Whitaker, 2006: 203-04). The Bergsonian response would be that the interruption of the electron's movement at the screen constitutes the extraction, from continuous time, of a distinct moment in the context of which the electron bears a definite value of position. 
Though Bohr believed classical objects such as measuring devices are exempt from the sway of the wave function, he could not explain why this should be the case. Even Schrödinger, despite having formulated the equation that governs the wave function, could not accept its ramifications. If an atom has a 50\% probability of decaying in a given timeframe, and the life of a cat depends on that atom not decaying, then clearly the wave function applies to the cat as much as the atom. Yet a cat cannot exist in a superposition of dead and alive (Whitaker, 2006: 237).

The fog clears when the problem is reconfigured in a temporal framework. Unlike its constituents, all of which can exist in either a superposition of potential states in continuous time or a determinate state in a distinct moment, a macroscopic object exists as such only on the basis of underlying determinate particles. Indeterminate particles and atoms do not add up to tangible objects. The time of a measuring device or a cat is a succession of distinct moments or space-time "snapshots" rather than the ongoing presence expressed by the smoothly propagating wave function.

We need not accept Wigner's dictum, also adopted by von Neumann, that the consciousness of the observer collapses the wave function, transforming superpositions into distinct values (Whitaker, 2006: 201). Nor do we need Girardi, Rimini and Weber's addition of a term to the Schrödinger equation in order to render, through artifice, nonlinear propagation of the wave function (Whitaker, 2006: 328). Nor do we need Bohm's attempt to eliminate wave function collapse altogether simply by invoking, from the get-go, particles with definite positions jostled about by quantum waves or, for that matter, Everett's claim that every possible outcome expressed in the wave function does in fact take place but that the observer sees only one version, mistaking it for "reality" (Zeh, 2010: 132).

Instead of seeking an answer outside Bohr's principle of complementarity, we need only deepen it. Bohr intended his principle to apply exclusively to the results of measurements, whether expressed as position or instant on the one hand or momentum or energy on the other. The first set of options entails "space-time coordination" while the second set enables a "claim of causality." We can either make an observation so as to place the quantum system in space and time or we can explain how the current state of the system causally relates to what preceded it. A deeper understanding, however, means recognizing measurement and the wave function as complementary perspectives onto the system, much as "particle" and "wave" are complementary descriptions of the results of measurements (Folse, 1985: 113-14).

This form of complementarity, in turn, expresses the twofold nature of time. In addition to wave propagation in continuous presence, we have instantiation on the basis of local interaction. To measure an electron is to cause it to interact with its large-scale environment, forcing it from the domain of continuous presence to that of momentary presence. In the context of a distinct moment it exhibits a precise value for the measured property. If we measure it again immediately afterward, the property will retain this value. Thus the instant is not, strictly speaking, instantaneous but carries a miniscule duration before passing into history, at which time the electron reverts to a superposition of values in accord with the wave function. The measurement postulate turns out to have been a placeholder for the instantiation postulate.

Instantiation is the physical process whereby indeterminate values resolve into determinate values. Measurement allows for well-defined values because instantiation is implicit in largescale processes, including measurement, since the constituents of large-scale objects are always interacting. The Schrödinger equation allows only for continued propagation of the wave function - making no provision for its collapse — because it represents only continuous 
time and leaves out the discontinuity of instantiation and the concomitant departure from the linear progress of the wave function.

The instantiation postulate clarifies the well known double slit experiment. If an electron is fired at a screen, and along the way the electron must negotiate a barrier with two narrow openings, so long as the electron remains undetected it remains in its default state - as determined by its wave function - and acts like a classical wave that passes through both slits. Granted, when it reaches the screen, it leaves a single mark in a seemingly random location. Over time, however, as more electrons are fired through the slits, the pattern that emerges on the screen is that of classical wave interference (Lewis, 2016: 5). By contrast, if detectors are placed at the slits, each electron loses its wave aspect, and the resulting pattern on the screen is a simple aggregate of marks beyond each slit. By interacting with the electron, the large-scale detector translates it from continuous time to a distinct moment consistent with a definite location.

An electron is a particle with determinate properties in the context of moment-tomoment time and an evolving superposition of potential values of properties in the context of continuous time. At no time are its properties both definite and indefinite. We have only a pair of temporally imposed complementary perspectives onto the quantum system.

\section{Memory as a Function of Temporal Complementarity}

The robustness of the principle of complementarity is revealed in the circuitous journey of David Bohm, who sought to get beyond Bohr's framework and directly express the reality of microphysical existence but wound up embracing the complementary notions of implicate and explicate order.

By proposing that an unmeasured quantum system includes particles with definite though hidden positions, Bohm reproduced all the predictions of orthodox quantum theory despite eliminating the special role of measurement and the need for wave function collapse (Lewis, 2016: 56). However, he also identified a deeper problem unaddressed in either orthodox theory or any of the alternative interpretations, including his own: continued reliance on the Cartesian grid, a coordinate system that describes an extended local order. While curvature can be introduced into the grid to accommodate general relativity, no amount of tweaking can make it suitable for quantum theory. This is due to the nonlocality inherent to "indivisible quantum processes that link different systems in an unanalysable way" (Bohm \& Hiley, 1993: 352). This problem was typically evaded, according to Bohm and Hiley (Bohm \& Hiley, 1993: 350), with the claim that quantum theory is based not on the physical world but on a mathematical world comprised of Hilbert space, an abstract space of potentially infinite dimensions, each one corresponding to a potential value of a property of a quantum system (Ney \& Albert, 2013: 6).

Insisting that physics describe the actual world, not a mathematical construct, Bohm devised the concept of implicate order so as to bring the mathematical and the physical into coherence (Bohm \& Hiley, 1993: 351). He illustrated his concept with the hologram. Whereas an ordinary photograph contains a one-to-one correspondence to a negative image on film and thus adheres to the Cartesian coordinate system, a hologram is a set of marks seemingly unrelated to the three dimensional picture projected from it. This is because the picture is distributed throughout the hologram, any portion of which can reproduce the picture in its entirety. Rather than simply project a pre-existent image for viewing, the holographic process explicates or "unfolds" a picture from information enfolded in the hologram (Bohm \& Hiley, 1993: 353-54). 
In addition to the spatial metaphor provided by the hologram, Bohm offered a temporal metaphor involving ink droplets processed through glycerin (Bohm \& Hiley, 1993: 358). The basic set-up is a cylindrical core that can be rotated within an outer cylinder. Between the cylinders is high viscosity glycerin. If we place an ink droplet onto the glycerin and then rotate the inner cylinder, the glycerin and the ink droplet swirl around it, causing the ink to disappear into the glycerin. If we then rotate the inner cylinder in the opposite direction, the ink droplet reconstitutes before our eyes. Thus the ink droplet, when no longer visible, is implicate or enfolded into the glycerin and becomes explicate again when the cylinder is rotated back.

Bohm also noted that ink droplets can be added to glycerin sequentially, each one enfolded into the glycerin before the next droplet is added. Once the last of the sequence is enfolded, the droplets reappear one at a time when the rotation is reversed. By rotating the cylinder at high speed, we see what appears to be a single steady dot. We can even make the dot move by enfolding droplets at sequentially different positions in the glycerin before rotating the cylinder in reverse at high speed. An electron, like the apparently enduring dot, takes on the appearance of an enduring particle on the basis of the rapidly repeating unfolding of implicate information. Bohm replaced the continuous trajectory of a determinate particle with a rapid succession of waves converging, at each instant, on a given point (Bohm \& Hiley, 1993: 359, 374).

To summarize, in response to the Einsteinian imperative to restore certainty to the microphysical, Bohm postulated a particle with a precise position even before measurement. However, he could not stop himself from taking the next step. Motivated by the need for "a notion of order for all our concepts, both mathematical and physical" (Bohm \& Hiley, 1993: 351 ), he proposed that the microphysical is the overlap of implicate and explicate orders, perhaps not realizing these orders are complementary in the exactly the sense that the wave function and the measuring device provide complementary views into a quantum system. Whether we call it implicate order or wave function, information periodically explicates or projects into determinate structure, i.e. matter.

Yet something of great value came out of Bohm's journey from point A to point A: the basis of memory in nature. As opposed to the artifice of storing data about the past, nature's memory is the continuation of past into present, that is, implicate endurance in the face of successive explications (Bohm \& Hiley, 1993: 382-83). By resetting with each measurement and evolving from this revised starting-point, the wave function abstracts whatever has explicated, in effect saving in outline form each tangible moment for future reference. Bohm's contribution was not so much to provide an alternative to Bohr as to demonstrate how memory can arise from the combination of wave mechanics and the quantum transition.

The wave function acknowledges neither future nor past. Indifferent to the direction of time, it describes the subtraction of superposed values in reverse time as readily as their addition in forward time. This is why, in the double slit experiment, the choice of whether or not to detect, say, a photon can be delayed until after it has passed the slits (Jacques et al, 2007: 966-968). Because the photon in its default state occupies continuous presence, it has no history distinct from its present. Thus, its passage through the slits is still present when the decision is later made to detect which slit it went through. The photon can be retraced to its arrival at the slits because its entire path remains present. By contrast, once a mark appears on a screen, it cannot later be made to disappear. Only indefinite presence, as revealed in the continuous wave function, is time-reversible.

According to Feynman's path integral method, nicknamed "sum over histories" by his mentor, Wheeler, every possible particle interaction in the double slit experiment exists in 
superposition (Halpern, 2017: 75, 82). The wave interference pattern on the screen is the summation of the possible histories of the particles. We might say the histories maintained in the ongoing presence of the wave-mechanical state ultimately wash ashore on a distinct moment. Ironically, Wheeler denied a fundamental reality to time on the grounds that "before" and "after" fail at the Planck length (Halpern, 2017: 199). What he overlooked is that the smallest distance in nature $\left(10^{-35} \mathrm{~cm}\right)$ corresponds to Planck time $\left(10^{-43}\right.$ seconds), an interval too brief to crystallize into a classical moment, leaving only the fundamental time of an ongoing present without beginning or end.

Since memory requires a "before" distinct from the present, the time-reversibility of wave propagation cannot by itself constitute memory. Nor is there natural memory in strictly tangible existence, only the artificial memory of stored data. True memory requires not only the unfolding of information into a determinate state - the instantiation that distinguishes momentary presence from the past - but the enfolding of that determinate state back into the ongoing informational order. The accumulation of increasingly complex information over time in the implicate order allows for the evolution of increasingly complex systems in the explicate order of classical existence.

\section{Evolution as a Function of Memory and Instability}

Though the uncertainty principle could just as well be called the ambiguity principle since the quantum system itself - not our understanding of it - contains the "uncertainty," Heisenberg's terminology is useful insofar as it points up a similarity between wave mechanics and thought. Like thought, which represents aspects of the world, wave propagation represents possible states of a quantum system. To think is to consider alternative interpretations of a given circumstance and alternative options regarding action. The collapse of the wave function into a definite state is no different in principle from the collapse of thought into behavior. Given that the calculation of possible outcomes innate to mental activity is also at the core of all material activity, we ought to consider if this resemblance is a homology as opposed to mere analogy, much as Darwin wondered if hands and hooves are homologous.

The failure of $20^{\text {th }}$ century theorists to take up the potential for quantum mechanics as the basis of a naturalized mentality might be due to Wigner and von Neumann's spurious claim that consciousness collapses the wave function, occluding the parallel between measurement selecting from possible states of a quantum system and consciousness selecting from possible responses to a given situation.

Could mentality be an evolved form of wave propagation? If so, what is the mechanism by which the wave function transformed from the guiding principle of atomic behavior to that of neural behavior? The answer, if there is one, might be found at the boundary of order and chaos in the unstable system. Instability reveals two properties, holism and memory, which provide a bridge from the quantum to the conscious.

An undisturbed quantum system is not a collection of determinate particles but the wave propagation of potential values of properties of the entire system. In contrast to a machine, where the parts merely comprise the whole, the parts of a quantum system express the whole. The whole is fundamental and the parts subsidiary.

As Schrödinger observed, implicit in quantum theory is that electrons become nonlocally "entangled" due to a history of interaction (Ney \& Albert, 2013: 17). Electrons that previously interacted are entangled in the sense that the values of their properties are correlated regardless of their distance. Thus, nonlocality implies not only holism but memory. The history of interaction between electrons remains present to them so long as they remain undisturbed 
by the larger-scale environment (such as a measuring device). Whereas artificial memory is strictly local, like finding the right data in a hard drive or the right passage in a book, natural memory is nonlocal. Once enfolded into implicate order, the past has no location. It can be accessed from anywhere.

Natural memory provides a means by which the regularities of matter might have emerged from the lawless chaos of the initial universe. Since our current understanding of physical law is based on current properties of matter and strengths of forces, how can it account for their appearance in the first place? Instead of timeless laws giving order to matter, the random explications of the very early universe would have set in motion tendencies such that subsequent explications would likely play out the same way. In this way electrons emerged as carriers of charge along with a tiny amount of mass while neutrons emerged as carriers of far greater mass but no charge. Whereas photons always fall into the same state as nearby photons, electrons are excluded from the same state of nearby electrons. Current physical law cannot explain the basis of these and many other curious properties of matter. Perhaps they resulted not from timeless law but habit. A habit once ingrained is easily mistaken for a law.

Prior to nucleosynthesis the cosmos was open to countless possibilities (Unger \& Smolin, 2015: 171). Once a stable set of particles and their respective properties were in place, however, cosmic evolution could proceed according to the deterministic and time-reversible principles of classical mechanics. Different combinations of various types of particles make for different types of atoms, which combine in predictable ways to make molecules and so on. Under the benign rule of mechanism, time is reduced from active presence to passive parameter, a sort of yardstick to keep track of the inevitable unfolding of pre-determined forms.

However, the Newtonian dream is interrupted with the reintroduction of indeterminism in the form of unstable systems, as described in the science of nonequilibrium thermodynamics. Over time, a gas is likely to arrive at equilibrium as concentrations of pressure and temperature smooth out. This is known as entropy. Far from equilibrium, however, gases or liquids are likely to encounter instability, which can trigger synchronized behavior. When water is sufficiently hotter than its environment, for instance, it spontaneously forms into convection cells that rapidly convey heat into the surrounding air. In one of the Belousov-Zhabotinski reactions, billions of molecules simultaneously turn blue, then red, then blue again. Many more coordinated reactions like this demonstrate that matter takes on new properties far from equilibrium (Prigogine, 1997: 67).

Though a quantum system is ordinarily under the rule of the wave function, measuring a property of the system forces it into a distinct moment, collapsing the deterministic wave function and leaving the system momentarily in a semi-random state. Likewise, when a nonequilibrium system hits a phase transition known as a bifurcation point, it either fails altogether or evolves into greater complexity. Even when all the relevant variables are known, the behavior of the system at a bifurcation point cannot be predicted. Like an explicating quantum system, the bifurcating chemical system is undetermined (Prigogine, 1997: 68).

Because a thermodynamic system irreversibly pursues equilibrium rather than following deterministic time-reversible laws, unlike in the case of a mechanical system its current state cannot be traced back to former states. Though systems that resist equilibrium with their environments are also beyond the reach of deterministic law, their behavior reveals a nonmechanistic "cyclical" recall (Schneider \& Sagan, 2005: 73). For instance, a system of Taylor vortices, which forms in a fluid between a pair of cylinders when the rotation of the inner cylinder reaches a certain speed, abruptly changes its number of vortices as the 
rate of rotation further increases, and these quantum-like jumps cannot be predicted without knowledge of the system's history (Schneider \& Sagan, 2005: 129).

Whereas ordinary thermodynamic systems follow the entropic path of least resistance to the most probable state, nonequilibrium systems break down environmental sources of energy in order to generate increasingly improbable structures (Schneider \& Sagan, 2005: 143). Possessed of both recall and goal-directed activity, complex systems that self-organize on the basis of gradient-reduction operate holistically.

The behavior of self-organized systems in no way follows from either the determinism of classical dynamics or the probabilism of statistical thermodynamics. Instead, something like the wave function is reinstated at the macroscopic level in the form of a wavelike distribution of possible trajectories (Prigogine, 1980: 249). Though the classical assumption would be that these wave packets merely represent ignorance of the actual state of individual particles, given Bohr's realization that ambiguity is objectively part of the quantum system under study (Jammer, 1974: 69), the same might be true in the case of self-organized systems. It seems the fuzziness inherent to both the wave function and the mind also resides in the chemical system that stubbornly resists equilibrium with its environment.

If the self-organized chemical system expresses an evolved form of wave propagation, so too does its living descendent. Every organism, down to the simplest bacterium, harnesses energy to maintain itself out of equilibrium with its environment. Yet we say an object is alive not because it is bounded and internally coherent - or even that it actively imports useful energy while dissipating entropy into its surroundings - but simply because it reproduces. In reality, the key factor that makes an organism alive is not its self-replicating DNA but its status as a self-referential whole (Schneider \& Sagan, 2005: 104). According to Mae-Wan Ho, the "structure of living organisms arises as the consequence of energy flow and is strongly reminiscent of the nonequilibrium phase transitions that can take place in physiochemical systems." Metabolism begins in complex chemical systems and evolves into the basis of biological systems (Schneider \& Sagan, 2005: 111, 236).

Just as life precedes genes, intelligence precedes brains. "Slime mold," as Schneider and Sagan point out, "has been shown to figure out and remember maze routes to get at food, and to split itself into two and rejoin along the shortest paths in mazes provided with two food sources" (Schneider \& Sagan, 2005: 155). As this physiological variant of superposition demonstrates, what really counts is not the accoutrements of higher biological systems but that, like the quantum system in its default state, the parts serve the whole rather than merely comprising it.

Self-organized systems extract energy from the environment by breaking down gradients in pressure or temperature. (Schneider \& Sagan, 2005: 85). Convection cells are powered by the energy unleashed from breaking down the temperature gradient between heated water and the surrounding air. Taylor vortices form spontaneously from a pressure gradient caused by rotation rate. Photosynthesis exploits the gradient between sunlight and the cool terrestrial environment. Whether chemical or biological, the pursuit of self-interest in the extraction of energy through gradient-reduction demonstrates self-existence and therefore at least the germ of mentality.

Whatever is influenced by its past, including its remote past, feeds into and in turn is nourished by implicate order. Like the first particles to emerge from primordial chaos, chains of chemical reactions spontaneously appearing in the early Earth enfolded into the background order and informed subsequent explications such that similar reaction chains became more likely. Without direction provided by implicate order, the emergence of life is exceedingly unlikely. 
Whereas an ordinary causal mechanism operates in the moment-to-moment time of explicate order, natural memory is the continued influence of past explications by way of ongoing implicate order. Memory is a function of the wholeness of past and present. Given our habit of thinking spatially, holism is typically regarded in terms of a system of objects across space. In this sense holism is the spatialization of memory, the application of the whole to a single moment across space. Whether temporal or spatial, holism is ultimately rooted in the indivisibility of ongoing presence, that is, in Bergson's duration.

"Every organism arises out of itself," wrote Schelling. "Every organic product carries the reason for its existence inside itself, for it is cause and effect of itself" (Gare, 2011: 52). How can a thing cause itself except in a continuous present? In the domain of distinct moments, effect must follow cause. The self-organized system - and the living system that evolves from it - expresses time as ongoing presence and not just successive moments.

In contrast to a distinct moment, which is present only relative to the moments preceding it, fundamentally time is presence without relation to anything external to it, that is, according to itself. Operating in absolute presence, wave propagation is at the root of self-existence and ultimately consciousness.

Hameroff and Penrose suggest that "consciousness occurs due to quantum vibrations in brain microtubules" and that each "objective reduction" of the wave function corresponds to a moment of consciousness (Hameroff, 2014: 126, 132). Yet the wave function directs not just individual particles but whole systems. An evolved form of wave propagation would coordinate the activities of an entire brain, not just particles embedded in brain cells. As atoms materialize wave-mechanical information, neural systems materialize higher-order information. This is not to deny the significance of microtubules in conscious states. However, consciousness itself cannot be contained in microtubules any more than an atom materially contains its wave function.

Whether a wave function explicating into matter or the evolved form of wave propagation we know as consciousness explicating into a pattern of neurotransmission, that which is ongoing and potential manifests in a determinate state in the context of a distinct moment. To be conscious is to sail on a sea of ambiguity in a continuous present informed by living history in pursuit of self-propagation. We are wave made flesh.

\section{Something from Nothing}

As Wheeler liked to ask in his later years, "How come existence?" (Halpern, 2017: 19). In other words, why is there as opposed to is not there?

The question implies that existence and nonexistence are mutually exclusive. To get beyond the conundrum we must recognize that these concepts are inseparable. While it would seem that by placing "not" in front of being we thereby negate it, in fact we merely contextualize it. Not-being is implicit in being. To have being without not-being is like having a planet without the space it occupies. Space or void is not a thing in itself but simply the background that makes the foreground possible.

From a temporal standpoint, being is a distinct present moment, and not-being is the same moment when it has passed. The gradient between them depends on discontinuous time. In continuous presence there is no such gradient, as the wave function gradually interweaves them in the form of a superposition of potential values. Only when the wave function collapses is there a distinction or gradient between being and not-being. The momentary present is the triumph of being. Its passage is the triumph of not-being. The most fundamental cycle of nature can be conceived as the collapse and restoration of the wave function or, 
alternatively, the emergence and collapse of the ontological gradient. Whereas collapse of the wave function yields matter, collapse of the ontological gradient yields information. In the context of the evolved or biological form of wave propagation, information is known as mind.

Though Dirac regarded a positron as a negative energy electron, Feynman conceived it as a temporally reversed electron (Muller, 2016: 242). Because ongoing presence does not admit of past or future, the undisturbed quantum system is indifferent to the arrow of time. Whether the wave function charts increasing or decreasing superposition of values depends on whether we take the electron's or the positron's point of view. As entropy demonstrates, only with successive instantiations does time take on a particular direction.

For the electron, the wave function gradually mixes being and not-being until the instantaneous collapse of superposition and restoration of the ontological gradient. For the positron, the wave function gradually sorts out being and not-being until the sudden collapse of the ontological gradient and the restoration of fully developed superposition. Like the convergence of sound waves with perfectly opposed amplitudes, the two processes cancel each other out. Something turns out to be nothing.

In addition, nothing turns out to be something. Directionality of time is revealed by not only entropy but also memory, by the accumulation of forms abstracted from successive explications. Simply by breaking the symmetry in favor of the electron's temporal arrow over the positron's, nature extracts something from nothing.

Like the wave function, pure thought admits of no preferred direction of time. Ideas seem to exist outside of time, and we can follow a train of thought in either direction, from $2+2=4$ to $4-2=2$. However, every time we act - every time the personal wave function collapses into definite action - we too extract something from nothing.

According to Bohm (Bohm, 1996: 113), even mass, the basis of our sense of material substance, decomposes upon examination into a pair of reciprocal qualities, inertia and gravitation, which turn out to be, in light of general relativity, the resistance to and generation of acceleration. What is left when they cancel each other out?

From the point of view of the explicate - the domain of definite properties - information seems to be nothing. For the implicate, the tangible existence resulting from explication is but a fleeting appearance. For each the other is nothing. Only in their union is there something.

Just as each new explication instantiates flowing presence, so too did the "big bang." Far from initiating time, cosmic ignition merely instantiated it. The meaning of the wave function is that the "nothing" at the heart of matter is not static but temporal, that is, animate. Life is no accident but cosmically embedded.

According to the well known anthropic principle, without precisely the right values of numerous constants of nature such as the mass of the electron, the strength of the binding force of the atom, the relative strengths of electromagnetism and gravity, etc., we would not be here to witness the world. Because the fundamental parameters were set very early in cosmic development, we must surmise that the universe, like a healthy newborn, came into existence in the one right way and not in any of the myriad wrong ways. Perhaps in both cases the knowledge of the right way to enter existence resulted from the many previous explications of similar systems. If so, not just this or that species of atom or mammal but also the universe as a whole is a product of memory and evolution. How many universes had to come and go before this one got it right? 


\section{Conclusion}

The rapid succession of explications of the wave function provides the basis for all sensorial experience (Bohm \& Hiley, 1993: 382). What we think is happening now is precisely what has been removed from the absolute present, set aside in a momentary present. The classical world is on a delay.

On the basis of observations of the human nervous system, Benjamin Libet discovered that human awareness is on a half second delay (Libet, 2004: 72). What we think is happening now in fact happened half a second ago. Given that classical existence is already on explicate delay, Libet's delay turns out to be old news. What difference does it make whether our sense of "now" is once or twice removed from ongoing presence, i.e. true now? Since the "stuff" of consciousness is not the brain's moment-to-moment existence but the continuous presence of mind, consciousness need not be bound to the extremely rapid recurrence of explicate matter. Our on-off cycle is not that of classical objects but that of waking and sleeping. So long as we remain awake, we are continuous in time. Though our sense of what constitutes the momentary present is mistaken, this mistaken sense is itself absolutely present. Precisely because presence is ongoing, a delayed sensation can always be re-presented in consciousness.

An electron is information in the context of ongoing presence and a determinate particle in the context of a distinct moment. Consciousness is mind in the ongoing present and neurotransmission in the momentary present. By pursuing a deeper understanding of complementarity instead of seeking to supplant it in favor of alternative interpretations of quantum mechanics, we find resolution of not only the quantum measurement problem but also the dualistic impasse that originally gave rise to it.

\section{References}

Albert, D.Z. (2015) After Physics. Harvard University Press.

Bergson, H. (1911) Matter and Memory. Swan Sonnenschein.

Bohm, D. (1996) The Special Theory of Relativity. Routledge.

Bohm, D. and Hiley, B. (1993) The Undivided Universe. Routledge.

Capek, M. (1971) Bergson and Modern Physics. D. Reidel.

Folse, H. (1985) The Philosophy of Niels Bohr: The Framework of Complementarity. Elsevier. Gare, A. (2011) From Kant to Schelling to Metaphysics: On the Way to Ecological Civilization. Cosmos and History: The Journal of Natural and Social Philosophy. Vol. 7, No. 2.

Halpern, P. (2017) The Quantum Labyrinth. Basic Books.

Hameroff, R. (2014) Consciousness, Microtubules, and "Orch-OR," a "Space-time” Odyssey. Journal of Consciousness Studies. Vol. 21, No. 3-4.

Jacques, V., Wu, E., Grosshans, F., Treussart, F., Grangier, P., Aspect A., Roch, J. (2007) Experimental realization of Wheeler's delayed-choice Gedanken Experiment. Science 315, 5814. 16 February 2007.

Jammer, M. (1974) The Philosophy of Quantum Mechanics. John Wiley \& Sons.

Lewis, P.J. (2016) Quantum Ontology. Oxford University Press.

Libet, B. (2004) Mind Time: The Temporal Factor in Consciousness. Harvard University Press.

Muller, R. (2016) Now: The Physics of Time. W.W. Norton.

Ney, A. and Albert, D.Z. (2013) The Wave Function. Oxford University Press.

Prigogine, I. (1980) From Being to Becoming. W.H. Freeman.

Prigogine, I. (1997) The End of Certainty. The Free Press. 
Schneider, E.D. and Sagan, D. (2005) Into the Cool: Energy Flow, Thermodynamics and Life. University of Chicago Press.

Unger, R.M. and Smolin, L. (2015) The Singular Universe and the Reality of Time. Cambridge University Press.

Whitaker, A. (2006) Einstein, Bohr and the Quantum Dilemma. Second Edition. Cambridge University Press.

Zeh, H.D. (2010) The Physical Basis of the Direction of Time. Springer-Verlag. 